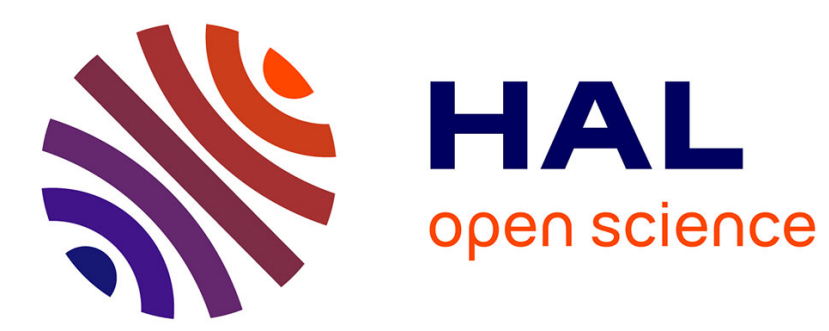

\title{
APPLICATIONS OF A FULLY VARIATIONAL METHOD FOR SOLVING ZERO ORDER THOMAS-FERMI EQUATIONS
}

Eric Suraud

\section{- To cite this version:}

Eric Suraud. APPLICATIONS OF A FULLY VARIATIONAL METHOD FOR SOLVING ZERO ORDER THOMAS-FERMI EQUATIONS. Workshop On Semiclassical Methods In Nuclear Physics, 1984, Grenoble, France. pp.C6-161-C6-166, 10.1051/jphyscol:1984619 . jpa-00224220

HAL Id: jpa-00224220

https://hal.science/jpa-00224220

Submitted on 1 Jan 1984

HAL is a multi-disciplinary open access archive for the deposit and dissemination of scientific research documents, whether they are published or not. The documents may come from teaching and research institutions in France or abroad, or from public or private research centers.
L'archive ouverte pluridisciplinaire HAL, est destinée au dépôt et à la diffusion de documents scientifiques de niveau recherche, publiés ou non, émanant des établissements d'enseignement et de recherche français ou étrangers, des laboratoires publics ou privés. 


\author{
E. Suraud \\ Division de Physique Théorique , Institut de Physique Nucléaire, \\ 91406 Orsay Cedex, France
}

\begin{abstract}
Résumé - Nous présentons quelques résultats obtenus dans un calcul Thomas-Fermi effectué sans resteindre l'espace variationnel et nous le comparons à ceux obtenus avec des fonctions d'essais et à ceux de la méthode du champ moyen, dans le cas de l'équation d'état de la matière dense.
\end{abstract}

Abstract - We present some results obtained in a Thomas-Fermi calculation without restriction of the variational space. We compare them to those obtained with trial functions and to the results of the mean field approximation, in the case of the equation of state of hot dense matter.

In the Thomas-Fermi type calculations, the nucleus is described with the help of the densities $\rho_{n, p}(r)$, by eliminating the kinetic and spin-orbit energy density terms in the energy functional. Various methods have been developed for this purpose. In most cases the variational parameters $\rho_{n, p}(r)$ are approximated by trial functions / // which destroy the self-consistency of the calculation. We have constructed a method of solution of the Thomas-Fermi equations without restriction of the variational space of the density profiles. We apply this method to calculations of the hot dense matter which occurs in the supernovae explosions $/ 2 /$. We show that the zero-order Thomas-Fermi method is a very good approximation to the Hartree-Fock results at high temperature, while, in contrast, the use of trial. Fermi functions gives a rather poor agreement with Hartree-Fock calculations.

\title{
Method
}

We minimize the free energy functional $F\left(N, Z, T, R_{c}\right)$ of a system containing $N$ neutrons and $Z$ protons inside a spherical box of rădius $R$ and at a temperature $\mathrm{T}$; the nucleon-nucleon interaction being a Skyrme type one. For this purpose we construct a series of profiles $\rho_{\alpha}^{(n)}(r)$ ( $\alpha$ labelling neutrons or protons) defined by

$$
\left.\rho_{\alpha}^{(n+1)}(r)=\rho_{\alpha}^{(n)}(r)\left(1-\lambda^{2}{ }_{\alpha}^{\left(F_{\alpha}^{\prime}(n)\right.}-\frac{1}{\int \rho_{\alpha}^{(n)} d \vec{r}} \int F_{\alpha}^{\prime(n)} \rho_{\alpha}^{(n)} d \vec{r}\right)\right)
$$

where $F_{\alpha}^{\prime}(n)$ is the functional derivative of $(n)$ the free energy with respect to the density $\rho_{\alpha}(r)$, calculated for $\rho_{n, p}(r) \equiv \rho_{n, p}(r)$, and $\lambda^{z}$ is a parameter of the method, similar to the imaginary time step $/ 3 /$.

The recursive relation (1) leads to the minimization of the free energy by means of successive first order variations. The technical details concerning this method have been described in previous publications $/ 4,5 /$.

In figure 1 we present a comparison between Hartree-Fock and Thomas-Fermi density profiles. The agreement is very good for the bulk parts while the description

\footnotetext{
*Laboratoire associé au C.N.R.S.
} 
of the surface is slightly different, due to the lowest order Thomas-Fermi approximation we use.
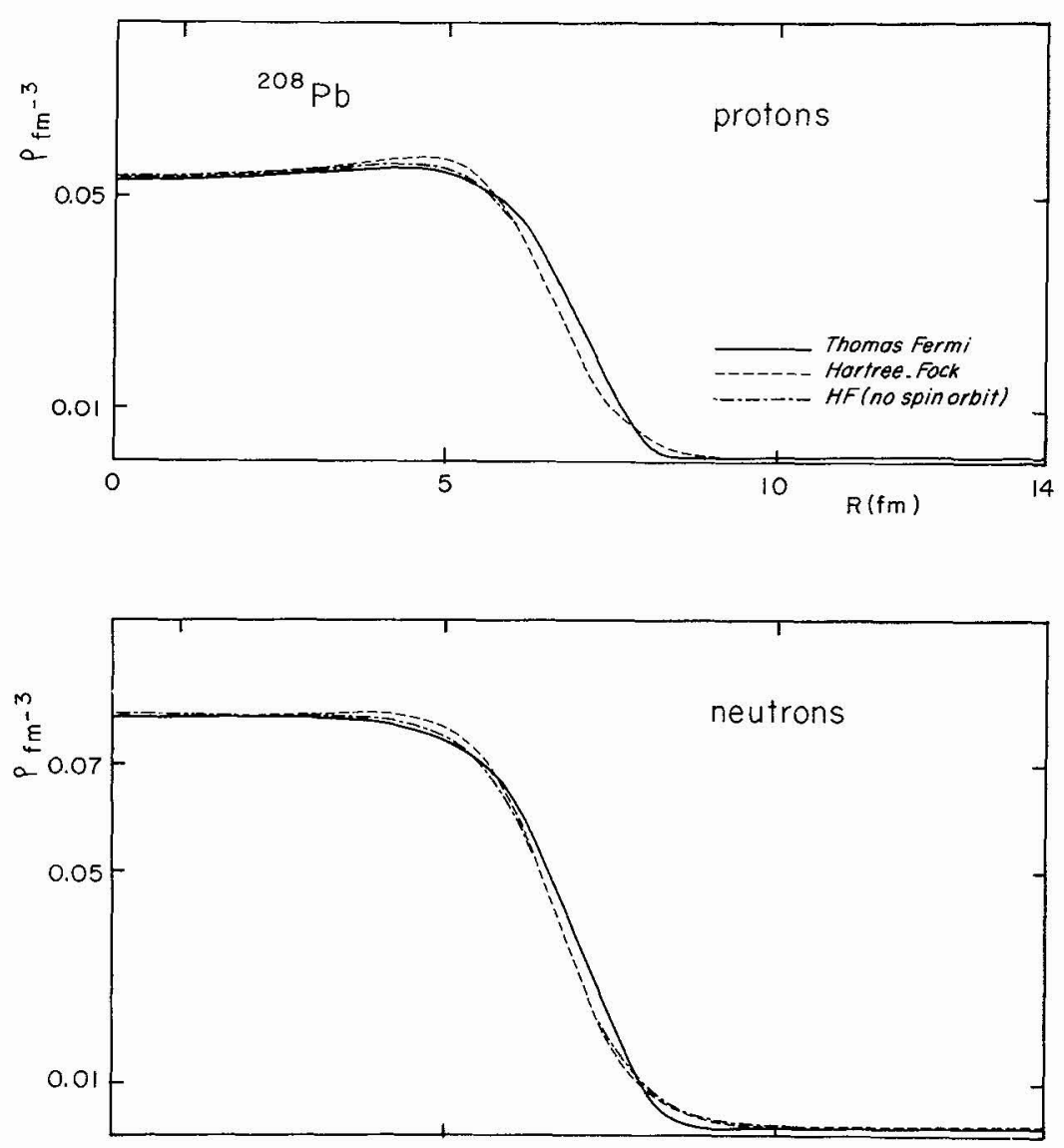

Fig.1 - Proton and neutron distributions obtained in lead 208 with interaction SIII in complete Thomas-Fermi and in Hartree-Fock calculations. The box size is $14 \mathrm{fm}$ and the temperature $6 \mathrm{MeV}$.

\section{Applications to the Equation of State}

The equation of state, which gives the pressure $P$ as a function of density $\rho$ and temperature $T$, is an important ingredient in the description of stellar collapse and supernovae explosions. For densities up to about nuclear density, the matter is a mixture of protons, neutrons, nuclei, electrons and neutrinos. 
The calculations are performed within a spherical Wigner-Seitz cell for a representative nucleus in equilibrium with the nucleon gas, electrons and neutrinos being included as free gases.

Various methods have been used to describe this exotic system, ranging from the bulk matter approximation /6/ and the Liquid drop model /7/ to more microscopic approaches as Thomas-Fermi $/ 8 /$ and Hartree-Fock calculations 19/. The mean field approximation gives the most reliable results but the numerical work involved becomes untractable at high temperature. The Thomas-Fermi method which is much simpler seems to be very adequate because the shell effects are washed out at the relevant temperatures $(T \sim 5 \mathrm{MeV}$ ) and because we are only interested in average quantities such as pressures and entropies.

In figure 2 we show the density profiles $\rho_{n}(r)$ and $\rho_{p}(r)$ calculated for a particular configuration of the equation of state labelled by the entropy per baryon $s=1$ and the lepton fraction $\gamma_{l}=0.35 / 10 /$. The results obtained with trial Fermi functions /11/ are in rather bad agreement with those of the mean field approximation $/ 9 /$. On the contrary our complete Thomas-Fermi calculation gives a satisfactory description of both the surface and the bulk parts, which shows the limitations of using an arbitrary restricted subspace for the density profiles $\rho_{n, p}(r)$.

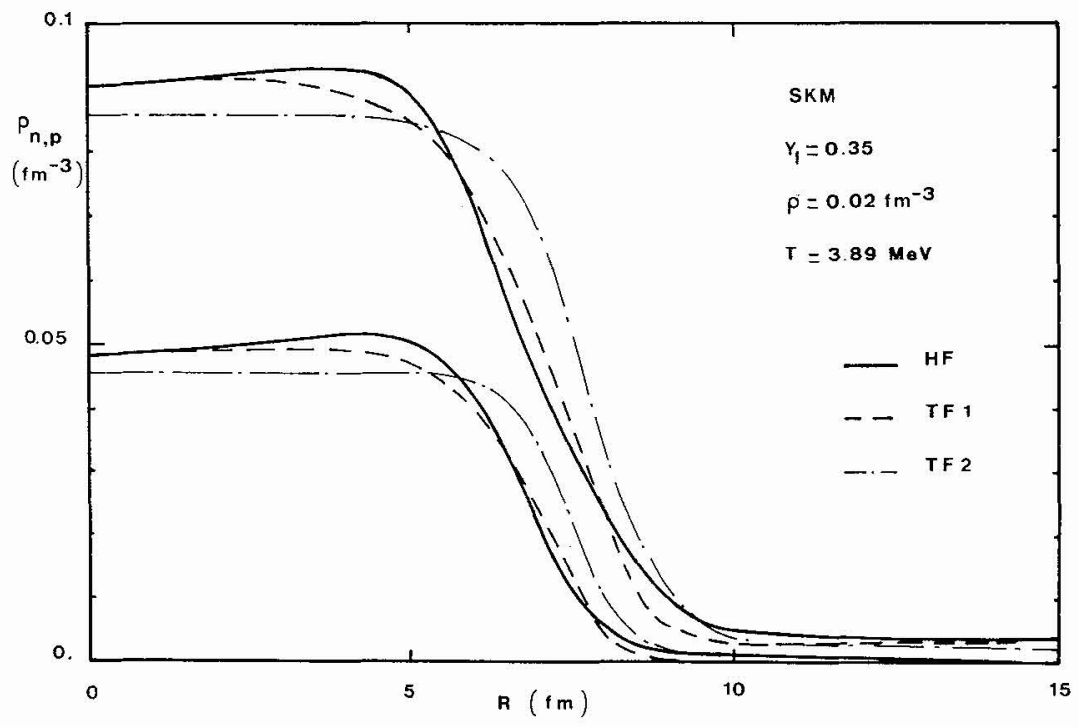

Fig. 2- Proton and neutron distributions in the particular configuration $\rho=0.02$ $\mathrm{fm}^{3}$ and $T=3.89 \mathrm{MeV}$ of the equation of state labelled by $\mathrm{s}=1$ and $Y_{\ell}=0.35$, for Hartree-Fock (full line (HF)), complete Thomas-Fermi (dashed line (TF1)) and Thomas-Fermi with trial functions (dashed-dotted line (TF2)) calculations. The interaction is SKM.

When the density $\rho$ reaches approximatively the nuclear matter density $\rho_{0}$ the nuclei dissolves into homogeneous matter. This transition stops the collapse because the adiabatic index $\gamma=\frac{\partial \operatorname{Ln} P}{\partial \operatorname{Ln} \rho}$ varies abruptly from less than $4 / 3$ (electronic 
pressure) to more than $5 / 3$ (nucleons pressure). This products pressure waves which generate the shock and the possible explosion of the outer shells of the star. The success or failure of the shock results from a tenuous energy balance and a crucial parameter appears to be the deviation of the mean adiabatic index $\gamma$ from $4 / 3$, for the densities up to $\rho_{0}$.

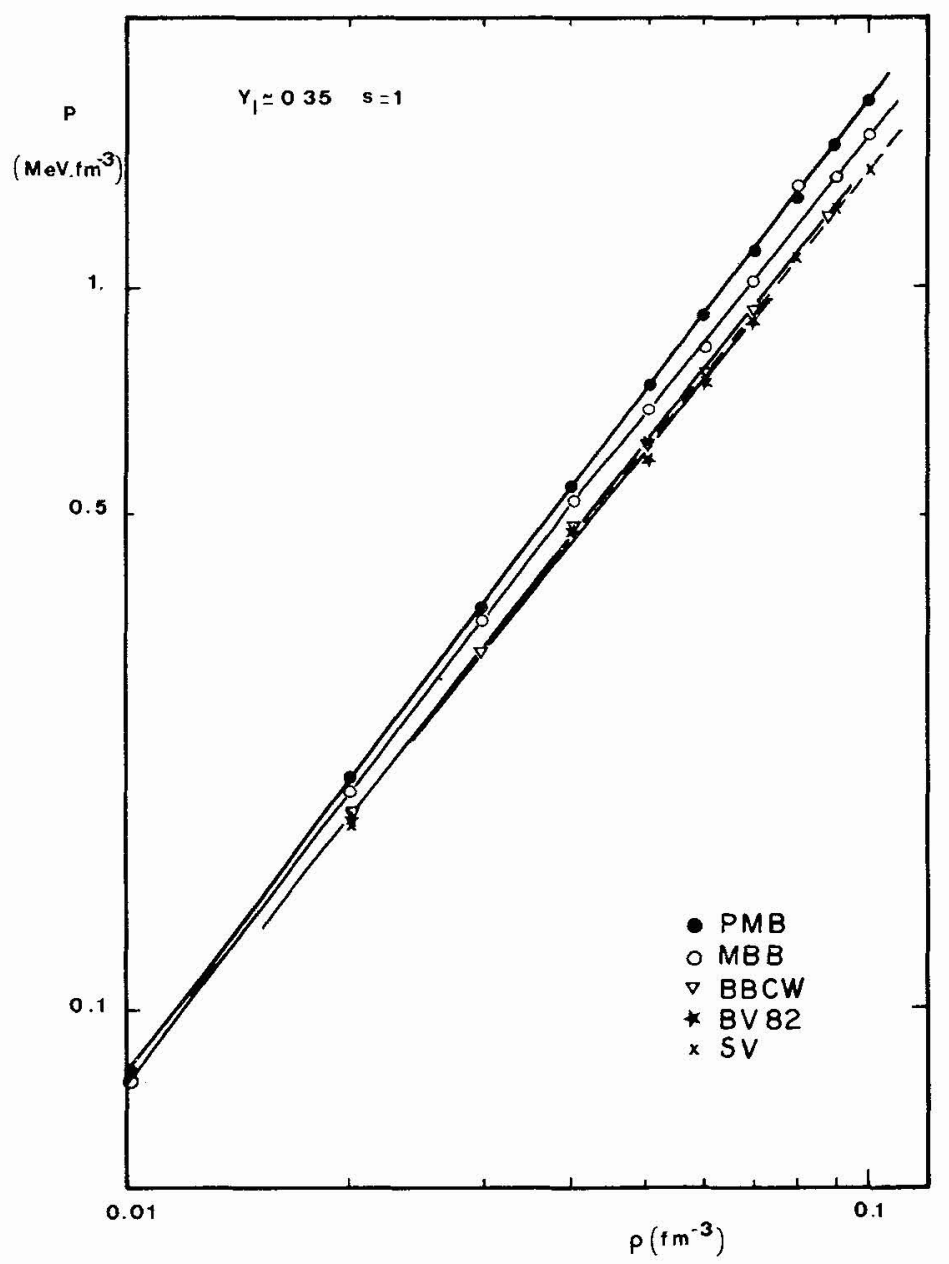

Fig.3 - Comparison of the equations of state $P(\rho)$ obtained in bulk matter approximation (PMB /14/), Liquid drop model (BBCW /15/), Thomas-Fermi with trial functions (MBB /8/), Hartree-Fock (BV $82 / 9 /$ ) and our complete ThomasFermi calculations (SV). The scales are logarithmic.

In $f$ igure 3 we present the equations of state obtained by diffenent methods $/ 6,7,8,9 /$ and we compare them to our results. The agreement is excellent between our Thomas-Fermi method and the Hartree-fock calculations. The other results are slightly different. To check the accuracy of these various approaches versus the mean field approximation / $9 /$ we consider the simple model of Yahil and Lattimer $/ 12,13 /$ who study a polytropic equation of state

$$
P \propto \rho^{\gamma}
$$


(in agreement with figure 3 ).

The authors estimate the energy of the shock in function of the adiabatic index $\gamma$, the electron fraction $Y_{e}$ and the density of transition $\rho_{\text {trans }}$ between "nuclei" and homogeneous matter

$$
E_{\text {shock }} \propto f(4 / 3-\gamma) \quad Y_{e}^{10 / 3} \rho_{\text {trans }}^{1 / 3}
$$

where $f$ is related to the mass of the inner core of the supernova.

In table 1 we present the term $f(4 / 3-\gamma)$ calculated for the equations of state of figure 3. The differences between the various approaches are now very obvious. In particular the $30 \%$ incercitude on the value of $f$ obtained in the equations of state of references $/ 8 /$ and $/ 15 /$ is too big to give reliable estimations of the shock energy.

\begin{tabular}{cccccc}
\hline Equation & PMB & BBCW & MBB & BV & SV \\
\hline$\gamma$ & 1.318 & 1.269 & 1.297 & 1.283 & 1.289 \\
\hline E/E & 1.53 & 0.66 & 1.30 & 1 & 1.12 \\
\hline
\end{tabular}

Table 1 - Influence of the adiabatic index $\gamma$ on the shock energy. The term $f(4 / 3-\gamma)($ eq.3) calculated for various equations of state (bulk matter approximation (PMB /14/), Liquid drop model (BBCW /15/), Thomas-Fermi with trial functions (MBB /8/), and our complete Thomas-Fermi (SV) is compared to the same term obtained in Hartree-Fock calculations (BV $/ 9 /$ ). The mean adiabatic index $\gamma$ is determined for $0.02 \mathrm{fm}^{-3} \leqslant p \leqslant 0.07 \mathrm{fm}^{-3}$.

\section{Conclusion}

We have shown that the use of trial Fermi functions in zero order Thomas-Fermi calculations may lead to bad approximations. On the contrary, the fully variational Thomas-Fermi method, we have developed, gives results in very good agreement with those of Hartree-Fock calculations. In particular, this method is very suitable for the stidy of hot dense matter and it would be worthwile to develop applications in this direction.

\section{References}

11/ BRACK M., Proceedings of the Workshop on Mathematical Methods in Nuclear Physics, Brussels, 1982.

121 VAUTHERIN D., Proceedings of the Workshop on Collapse and Numerical Relativity, Toulouse, France, November 7-11, 1983.

13/ DAVIES K.T.R. et al., Nucl. Phys, A342 (1980) 111.

14/ SURAUD E. and VAUTHERIN D., to be published in Physics Letters B.

15/ SURAUD E., Proceedings of the Workshop on Collapse and Numerical Relativity, Toulouse, France, November 7-11, 1983.

161 LAMB D.Q., LATTIMER J.M., PETHICK C.J. and RAVENHALL D.G., Nucl. Phys. A360 (1981) 459 . 
17/ LAMB D.Q., LATTIMER J.M., PETHICK C.J. and RAVENHALL D.G., Phys. Rev. Lett. 41 (1978) 1623.

18/ Marcos S., Barranco M. and BuCHLER J.R., Nucl. Phys. A381 (1982) 507.

19/ BONCHE P. and VAUTHERIN D., Astron. and Astrophys. 112 (1982) 268.

/10/ BETHE H.A., BROWN G.E., APPLEGATE J. and LATTIMER J.M., Nucl. Phys. A324 (1979) 487.

111/ HARTMANN D., El EID M.F. and BARRANCO M., submitted to Astronomy and Astrophysics.

112/ YAHIL A. and LATTIMER J.M., Proceedings of NATO Advanced Study Institute on Supernovae, Cambridge, England, June 29-July 10, 1981.

/13/ YAHIL A., Ap.J. 265 (1983) 1047.

114/ PI M., BARRANCO M. and MARCOS S., Il Nuovo Cimento 75A (1983) 100.

115/ BETHE H.A., BROWN G.E., COOPERSTEIN J. and WILSON J.R., Nucl. Phys. A403 (1983) 625. 